\title{
Composición química y evaluación de degradabilidad de residuos tóxicos de un núcleo ensilado a base de desechos de clavel
}

\section{Chemical composition and degradability study of toxic residues in cores silage in carnation waste}

Néstor Dairo Roberto López ${ }^{1}$

\begin{abstract}
Resumen
Se determinó la composición química y se cuantificaron los niveles de residuos tóxicos en núcleos ensilados de desechos de clavel (Dianthus caryophillus); los desechos de clavel fueron tomados del cuarto de poscosecha, y se enviaron muestras de clavel fresco al laboratorio de nutrición animal y al laboratorio de toxicología; posteriormente fueron mezclados con otros subproductos en los siguientes porcentajes: desechos de clavel, 50\%; gallinaza, 30\%; harina de alfalfa, 15\%; melaza, 5\%, y sal mineralizada, $1 \%$; con esta mezcla se hicieron microsilos para tomar muestras de éstos a los 0, 15 y 30 días de fermentación, las cuales se enviaron a los laboratorios. Los niveles de residualidad presentes en el núcleo ensilado luego de 30 días de fermentación mostraron una notoria reducción de porcentaje de todos los plaguicidas encontrados en la muestra de clavel fresco; algunos de estos plaguicidas tuvieron un mayor porcentaje de reducción que otros; este hecho podría evidenciar un posible efecto de las reacciones bioquímicas y microbiológicas que ocurren durante el proceso de ensilaje, que conducirían a la biotransformación o biodegradación de los compuestos encontrados. Por otra parte, el subproducto del clavel ensilado
\end{abstract}

\begin{abstract}
It was determined the chemical composition and quantified the levels of toxic waste in cores silage in carnation waste (Dianthus caryophillus), the carnation waste were taken from the postharvest room and sent samples of fresh carnation to the animal nutrition laboratory and toxicology laboratory, then were mixed with other products in the percentages: $50 \%$ carnation waste, 30\% chicken manure, $15 \%$ alfalfa meal, 5\% molasses, and $1 \%$ mineralized salt; with this mixture were made microsilos and took samples of these at 0 , 15 and 30 days of fermentation and were sent to laboratories. The residual levels present in the silage core after 30 days of fermentation showed a marked reduction in percentage of all pesticides found in the sample of fresh carnation. Some of these pesticides had a higher reduction percentage than others; this might reveal a possible effect of biochemical and microbiological reactions that occur during the ensiling process that would carry to the biotransformation or biodegradation of the compounds. Moreover, the by-product of silage carnations presented dry matter content of $52.8 \%$ higher than the fresh with $28.6 \%$. The protein fraction was decreased by the silage process up to $13.6 \%$ versus $16.7 \%$ in fresh. The ashes decrease
\end{abstract}

Médico Veterinario Zootecnista. Universidad Pedagógica y Tecnológica de Colombia. Tunja.

e-mail nestor.roberto@uptc.edu.co 
presentó un contenido en materia seca del 52,8\% mayor al fresco, con un $28.6 \%$. La fracción proteica se vio disminuida por el proceso de ensilado hasta el $13,6 \%$ frente al $16,7 \%$ en fresco. Las cenizas disminuyeron en casi dos puntos, y la grasa aumentó considerablemente, casi en un punto; la relación entre fibra en detergente neutro y fibra en detergente ácido aumentó.

Palabras clave: Residuos tóxicos, Proteína cruda, Ensilaje, Plaguicidas, Cromatografía. (Fuente: USDA) by nearly two points and the fat increased considerably almost in a point, the relation neutral detergent fiber and acid detergent fiber increased.

Key words: Toxic waste, Crude protein, Silage, Pesticides, Chromatography (Source: USDA) 


\section{Introducción}

Colombia está posesionada como el segundo productor mundial de clavel, con cerca de 2200 ha sembradas; aproximadamente el $10 \%$ de la producción de clavel del país se concentra en el departamento de Boyacá. La producción de desechos de clavel de cada hectárea mes Ilega a los $1500 \mathrm{~kg}$., el $10 \%$ de los cuales es utilizado en compostaje, y el $90 \%$ restante es utilizado clandestinamente en la alimentación animal, lo cual está prohibido, puesto que vacas lactantes alimentadas con desechos de clavel producen leche contaminada con residuos de plaguicidas (Aponte y Alfonso, 2008). Cerón (1993) enuncia la problemática de los residuos de plaguicidas con la utilización de desechos de clavel en alimentación animal.

Con este trabajo se buscan determinar la calidad nutricional de los desechos de clavel tanto en fresco como incluidos en un núcleo ensilado; de igual manera, se busca evaluar el comportamiento de los residuos de plaguicidas presentes en los desechos de clavel al ser sometidos a la actividad microbiana del proceso de fermentativo del ensilaje.

En busca de nuevas alternativas de nutrición vemos en el desecho de clavel una alternativa de alimentación animal, sin embargo, se debe tener en cuenta la existencia de residuos de plaguicidas en éstos (De-Prado y Osuna, 1999; Malagón, 2005); por esta razón se busca con prácticas zootécnicas, conseguir la inocuidad del producto, para no poner en riesgo la salud animal y, por consiguiente, la salud humana. Así, se hace necesario determinar la calidad nutricional de los desechos de clavel tanto frescos como mezclados en un núcleo ensilado; de igual manera, se busca detectar y cuantificar los niveles de residuos tóxicos presentes en este desecho.

\section{Materiales y Métodos}

El núcleo ensilado se elaboró en la finca Ruata, vereda Caitoque, en el municipio de Sora, ubicado a $19 \mathrm{~km}$. de Tunja; se encuentra entre los pisos térmicos frío y páramo, cuya temperatura oscila entre 10 y $14{ }^{\circ} \mathrm{C}$, a una altitud de $2650 \mathrm{msnm}$ (Alcaldía de Sora, 2012). Los desechos de clavel, materia prima de los núcleos ensilados, eran compuestos de hojas, flores y tallos, y fueron adquiridos de la empresa agrícola El Cactus S.A, ubicada en el municipio de Toca, a 2517 msnm.
A los cero días de salida de los desechos de clavel del cuarto de poscosecha se tomaron 40 $\mathrm{kg}$. en bolsas plásticas; estos desechos fueron transportados en coche a la finca Ruata, donde están los demás materiales para la elaboración del núcleo ensilado; se elaboraron seis microsilos; de esta manera se evitó destapar un microsilo por más de una vez para la toma de muestras. Los microsilos se elaboraron de la siguiente manera: $30 \mathrm{~kg}$. (50\%) de desecho de clavel, $18 \mathrm{~kg} .(30 \%)$ de gallinaza, $8.4 \mathrm{~kg}$. (14\%) de harina de alfalfa, $3 \mathrm{~kg}$. $(5 \%)$ de melaza y $0,6 \mathrm{~kg} .(1 \%)$ de sal mineralizada; posteriormente, el clavel y la gallinaza se molieron para su homogeneización de mezclado. Una vez molidos los materiales, se mezclaron manualmente; primero se mezcló la gallinaza, la harina de alfalfa y la sal mineralizada; después se agregaron los desechos de clavel, mezclando hasta homogeneizar la mezcla. Por último se agregó la melaza, mezclando fuertemente para evitar compactaciones. La mezcla obtenida registró una humedad entre el 20 y el 30\%; para garantizar el porcentaje de humedad se realizó la prueba de puño. Finalmente, el empaque al vacio se realizó manualmente en bolsas de polietileno de calibre 2 mm, y con una capacidad de 10 kilos.

A los cero días se tomaron de 400 a 600 g. de desechos de clavel fresco directamente del lugar donde son dispuestos en el predio San José de la agrícola El Cactus S.A.; se colocaron en bolsas de polietileno debidamente rotuladas, y de inmediato fueron enviados al laboratorio Nacional de Insumos Agropecuarios (LANIA) y al laboratorio de nutrición animal de la Uptc. Después de elaborado el ensilaje se tomaron muestras de éstos a los 0,15 y 30 días de fermentación; para esto se tomaron de 10 a 15 submuestras a diferentes frentes de cada microsilo por evaluar, y luego se mezclaron. De esta porción representativa se tomaron 1000 gramos y se empacaron en bolsas de polietileno de sello hermético para llevarlas de inmediato al Laboratorio de Nutrición Animal de la Universidad Pedagógica y Tecnológica de Colombia, donde fueron analizadas por el método de Weende o análisis proximal para determinar: \% materia seca, $\%$ cenizas, \% fibra detergente neutro, \% de fibra en detergente ácido, \% proteína y grasa.

De igual manera, se tomaron muestras de ensilaje a los 0,15 y 30 días de fermentación, las cuales fueron enviadas al laboratorio de toxicología del Instituto Colombiano Agropecuario (ICA) y al Laboratorio Nacional de Insumos Agrícolas (LANIA), y luego analizadas por el método de cromatografía de gases 


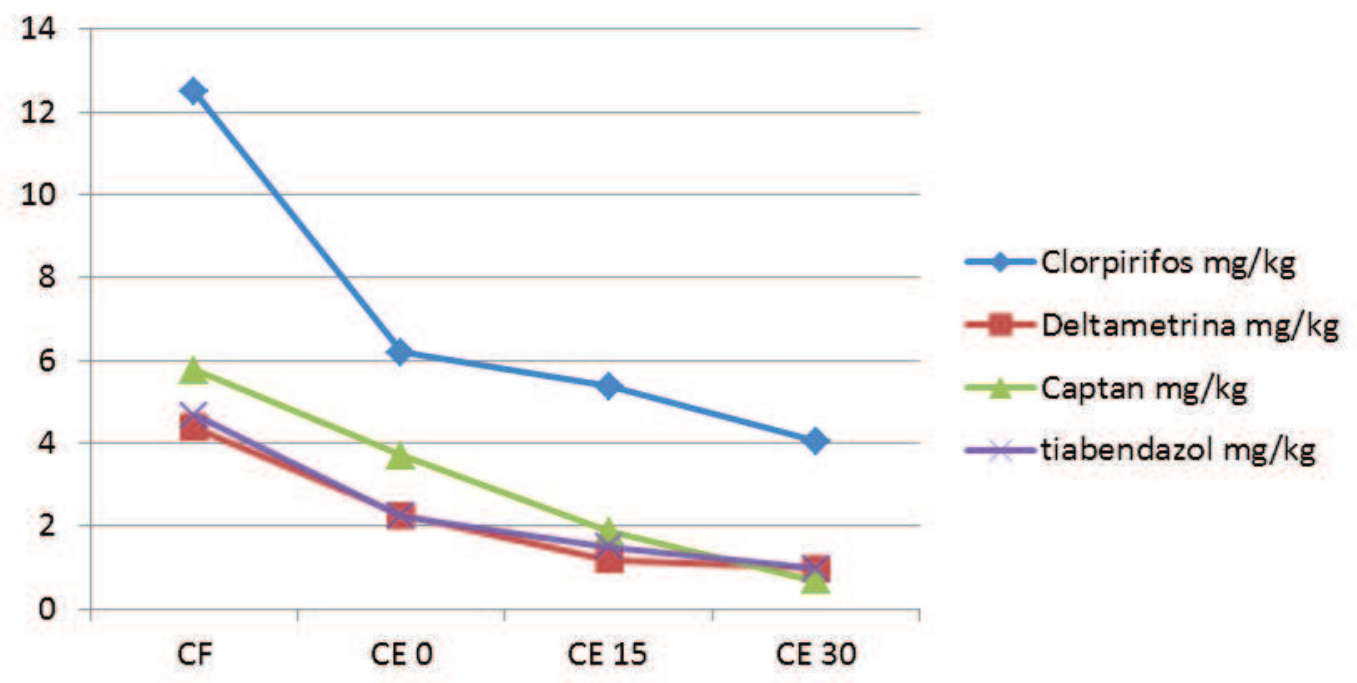

Figura 1. Comportamiento de los residuos de plaguicidas según el tiempo de fermentación.

con detectores de captura de electrones y fotométrico de Ilama para detectar y cuantificar residuos tóxicos de plaguicidas a base de organoclorados, organofosforados, piretroides, N-Metil carbamatos, Captan, clorotalonil y tiabendazol. Se detectaron y cuantificaran los anteriores grupos químicos y principios activos, porque hacen parte del control químico utilizado en la finca para la sanidad del cultivo. Algunos de estos plaguicidas son utilizados 15 días antes de la salida de la flor al mercado.
Cuando se obtuvieron todos los resultados se realizó un diseño completamente al azar con igual número de unidades por tratamiento, puesto que es fácil de planear y flexible en cuanto el número de tratamientos y repeticiones, utilizando un análisis de varianza (ANOVA) de un solo factor, que en este caso es el tiempo de fermentación de cada muestreo (fresco, 0, 15 y 30 días). Las diferencias estadísticas se hallaran por medio de una prueba de rango múltiple de Tukey.

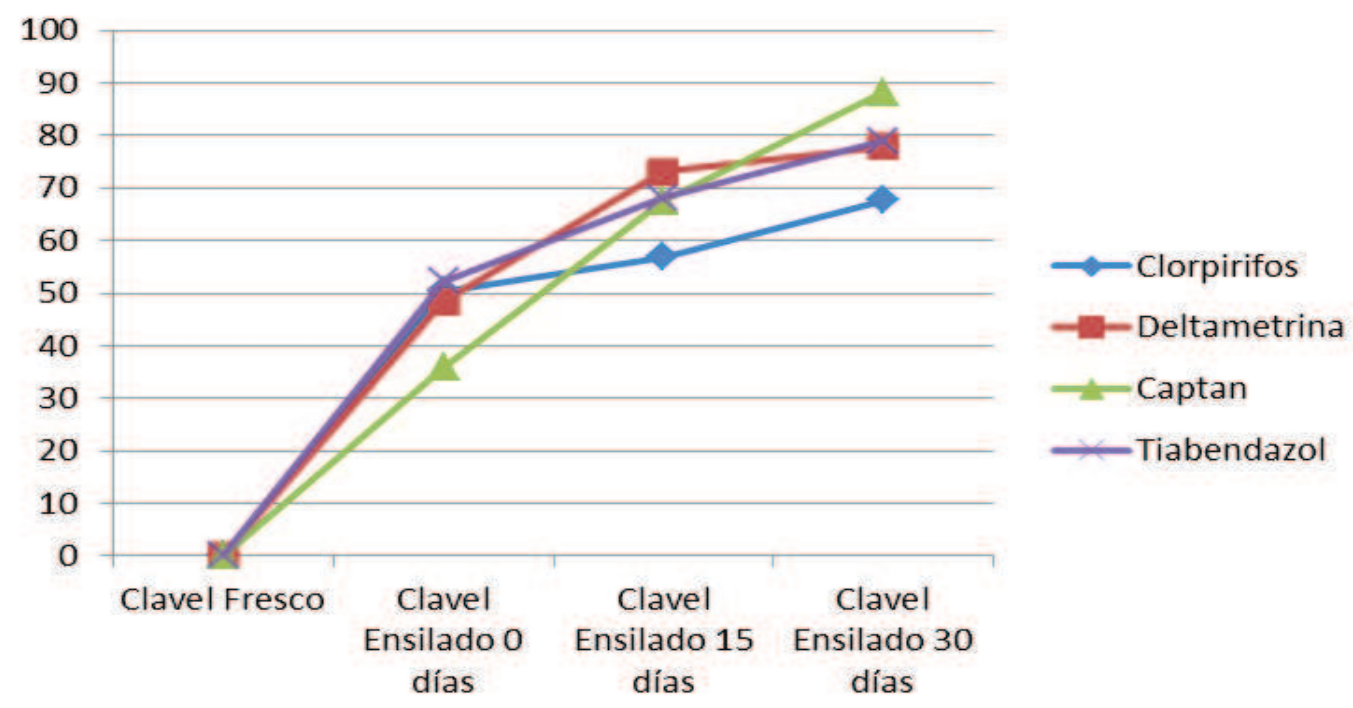

Grafica 2. Porcentaje de degradabilidad de los plaguicidas encontrados en las muestras estudiadas. 


\section{Resultados y Discusión}

Los residuos de plaguicidas de clorpirifos, deltametrina, Captan y tiabendazol se muestran en la gráfica 1, comparados con el tiempo de fermentación. Como se esperaba, en las muestras enviadas se encontraron residuos de plaguicidas. Los desechos de clavel fresco presentaron residualidad de todos los compuestos, excepto de N-metil carbamatos y clorotalonil; todas las residualidades presentes se deben a las aplicaciones de estos plaguicidas durante las prácticas modernas desarrolladas en el proceso de cultivo de clavel. Puesto que las muestras de clavel para el estudio fueron tomadas en época de verano, la cantidad de residuos de insecticidas aumentan, esto porque en verano la incidencia de plagas aumenta en un alto porcentaje.

Los niveles de residualidad presentes en el núcleo ensilado luego de 30 días de fermentación mostraron una notoria reducción de todos los plaguicidas encontrados en la muestra de clavel fresco. En la gráfica 2 se muestra que algunos de estos plaguicidas tuvieron un mayor porcentaje de reducción que otros; este hecho podría evidenciar un posible efecto de las reacciones bioquímicas y microbiológicas que ocurren durante el proceso de ensilaje, que conducirían a la biotransformación o biodegradación de los compuestos encontrados. Moorman (1990) argumenta que los microorganismos presentes en el suelo requieren de un proceso de adaptación para poder generar procesos de degradación de plaguicidas, presentándose una correlación entre la concentración y estructura química del plaguicida en el ambiente y la cinética de la degradación. Linkfield y cols. (1989), citados por Moorman (1990), reportan períodos de adaptación a una población microbial de 20 a más de 170 días en condiciones anaeróbicas.

No existe una normativa sobre límites máximos de residuos (LMR) para flores de corte, por lo tanto, es difícil tener una referencia válida sobre los resultados en clavel; sin embargo, para el estudio del caso se tomó la legislación colombiana y la legislación española para hacer comparaciones directas de los límites máximos de residuos de plaguicidas LMR en piensos o forrajes para la alimentación animal, y al no encontrar LMR para forrajes nos remitimos a niveles permisibles para alimentos humanos. Según la legislación colombiana, los niveles de residuos de clorpirifos en paja y forraje seco de trigo es de 5 $\mathrm{mg} \cdot \mathrm{kg}^{-1}$, en el producto final del ensilado tenemos un nivel de clorpirifos de $4.045 \mathrm{mg} \cdot \mathrm{kg}^{-1}$; esto indica que para este estudio estos niveles están dentro de los permisibles. Si tomamos la legislación española, el núcleo ensilado está muy lejos de los niveles permisibles en España, porque el LMR allí es 0.05 $\mathrm{mg} \cdot \mathrm{kg}^{-1}$.

En cuanto a la deltametrina, el LMR en tomate es de $0.3 \mathrm{mg} \cdot \mathrm{kg}^{-1}$ en la legislación colombiana; en la legislación española es de $0,5 \mathrm{mg} \cdot \mathrm{kg}^{-1}$ para alfalfa y leguminosas; en el núcleo ensilado, a los 30 días de fermentación contiene residuos de deltametrina que ascienden a los $0,97 \mathrm{mg} \cdot \mathrm{kg}^{-1}$, lo cual indica que este producto no está dentro de los niveles admisibles para la alimentación animal.

El Captan, ampliamente utilizado en la producción de tomate en Colombia, cuyo LMR es de 15,0 $\mathrm{mg} \cdot \mathrm{kg}^{-1}$, está muy por encima de los resultados obtenidos en las muestras de clavel ensilado a los 30 días de fermentación, cuyo valor es $0,68 \mathrm{mg} \cdot \mathrm{kg}^{-1}$; en cuanto a este fungicida, se puede decir que los niveles son permisibles para alimentación animal.

Según la legislación colombiana, en el cultivo de mango los LMR de tiabendazol son de 5.0 Po $\mathrm{mg} \cdot \mathrm{kg}^{-1}$ (Po: el LMR incorpora el tratamiento del producto después de la cosecha); por consiguiente, el ensilaje a los 30 días de fermentación es admisible para la alimentación animal porque tiene una cantidad de $0,98 \mathrm{mg} \cdot \mathrm{kg}^{-1}$.

Hay que tener en cuenta que los niveles de cada uno de los plaguicidas presentes en los desechos de clavel están directamente relacionados a las prácticas agrícolas realizadas días antes de la cosecha de la flor, puesto que cada asistente técnico tiene diferentes protocolos de control de plagas y enfermedades según variables como tiempo, humedad relativa, porcentaje de infestación y días para la cosecha (Hard y Araya, 1996).

Tabla 1. Clorpirifos según el tiempo de fermentación del núcleo ensilado.

\begin{tabular}{|c|c|c|}
\hline Muestra & $\begin{array}{c}\text { Clorpirifos } \\
\mathrm{mg}^{\prime} \mathrm{kg}^{-1}\end{array}$ & $\begin{array}{c}\% \text { de } \\
\text { degradabilidad }\end{array}$ \\
\hline Clavel Fresco & $12,48 \mathrm{C}$ & 0 \\
\hline $\begin{array}{c}\text { Clavel Ensilado } \\
0 \text { días }\end{array}$ & $6,19 b$ & 50,40 \\
\hline $\begin{array}{c}\text { Clavel Ensilado } \\
15 \text { días }\end{array}$ & $5,38 \mathrm{ab}$ & 56,89 \\
\hline $\begin{array}{c}\text { Clavel Ensilado } \\
30 \text { días }\end{array}$ & $4,04 \mathrm{a}$ & 67,62 \\
\hline
\end{tabular}

Letras diferentes en la columna, presentan diferencias estadísticamente significativas $(p<0,05)$ 
En el caso del clorpirifos, se presentan diferencias estadísticas significativas $(p<0,05)$ entre el clavel fresco $\left(12,48 \mathrm{mg} \cdot \mathrm{kg}^{-1}\right)$, el clavel ensilado a los cero días $\left(6,19 \mathrm{mg} \cdot \mathrm{kg}^{-1}\right)$ y el clavel ensilado a los treinta días $\left(4,04 \mathrm{mg} \cdot \mathrm{kg}^{-1}\right)$; entre los días 0 y 15 de fermentación no hay diferencias estadísticas significativas.

Tabla 2. Deltametrina según el tiempo de fermentación del núcleo ensilado.

\begin{tabular}{|c|c|c|}
\hline Muestra & $\begin{array}{c}\text { Deltametrina } \\
\mathbf{~} \mathbf{m} \cdot \mathbf{k g}^{-\mathbf{1}}\end{array}$ & $\begin{array}{c}\text { \% de } \\
\text { degradabilidad }\end{array}$ \\
\hline Clavel Fresco & $4,38 \mathrm{c}$ & 0 \\
\hline $\begin{array}{c}\text { Clavel Ensilado } \\
\text { 0 días }\end{array}$ & $2,26 \mathrm{~b}$ & 48,40 \\
\hline $\begin{array}{c}\text { Clavel Ensilado } \\
\text { 15 días }\end{array}$ & $1,18 \mathrm{a}$ & 73,05 \\
\hline $\begin{array}{c}\text { Clavel Ensilado } \\
30 \text { días }\end{array}$ & $0,97 \mathrm{a}$ & 77,85 \\
\hline
\end{tabular}

Letras diferentes en la columna, presentan diferencias estadísticamente significativas $(p<0,05)$

La Deltametrina tiene diferencias estadísticas significativas $(p<0,05)$ entre el clavel fresco $(4,38$ $\left.\mathrm{mg} \cdot \mathrm{kg}^{-1}\right)$, el clavel ensilado a los cero días $(2,26$ $\left.\mathrm{mg} \cdot \mathrm{kg}^{-1}\right)$ y el clavel ensilado a los 15 días $(1,18$ $\left.\mathrm{mg} \cdot \mathrm{kg}^{-1}\right)$; esto indica que en la primera mitad de la prueba fue cuando las concentraciones de residuos se rebajaron en mayor volumen.

Entre los 15 y 30 días de fermentación del ensilaje no hay diferencias estadísticas significativas ( $p>0,05)$; esto nos dice que sería lo mismo brindarle el alimento a un animal en cualquiera de los dos momentos de fermentación, pero hay que tener en cuenta que entre los 15 y 30 días si hubo una rebaja de residuos de deltametrina en el forraje, entonces lo más conveniente sería dar para la alimentación a los 30 días de fermentación.

Tabla 3. Captan según el tiempo de fermentación del núcleo ensilado.

\begin{tabular}{|c|c|c|}
\hline Muestra & $\begin{array}{c}\text { Captan } \\
\mathbf{m g} \mathbf{k g}^{-1}\end{array}$ & $\begin{array}{c}\text { \% de } \\
\text { degradabilidad }\end{array}$ \\
\hline Clavel Fresco & $5,76 \mathrm{~d}$ & 0 \\
\hline $\begin{array}{c}\text { Clavel Ensilado 0 } \\
\text { días }\end{array}$ & $3,69 \mathrm{c}$ & 35,93 \\
\hline $\begin{array}{c}\text { Clavel Ensilado } \\
\text { 15 días }\end{array}$ & $1,87 \mathrm{~b}$ & 67,53 \\
\hline $\begin{array}{c}\text { Clavel Ensilado } \\
\text { 30 días }\end{array}$ & $0,68 \mathrm{a}$ & 88,19 \\
\hline
\end{tabular}

Letras diferentes en la columna, presentan diferencias estadísticamente significativas $(p<0,05)$
El Captan, a diferencia de todos los compuestos cuantificados, presenta diferencias estadísticas durante todo el proceso fermentativo, siendo el día 30 cuando los niveles de residuos tóxicos son menores, al igual que en los demás plaguicidas; esto es a causa de las propiedades fisicoquímicas propias del Captan.

Tabla 4. Tiabendazol según el tiempo de fermentación del núcleo ensilado.

\begin{tabular}{|c|c|c|}
\hline Muestra & $\begin{array}{c}\text { Tiabendazol } \\
\mathbf{m g} \cdot \mathbf{k g}^{-1}\end{array}$ & $\begin{array}{c}\text { \% de } \\
\text { degradabilidad }\end{array}$ \\
\hline Clavel Fresco & $4,67 \mathrm{c}$ & 0 \\
\hline $\begin{array}{c}\text { Clavel Ensilado } \\
\text { 0 días }\end{array}$ & $2,23 \mathrm{~b}$ & 52,24 \\
\hline $\begin{array}{c}\text { Clavel Ensilado } \\
\text { 15 días }\end{array}$ & $1,49 \mathrm{a}$ & 68,09 \\
\hline $\begin{array}{c}\text { Clavel Ensilado } \\
\text { 30 días }\end{array}$ & $0,98 \mathrm{a}$ & 79,01 \\
\hline
\end{tabular}

Letras diferentes en la columna, presentan diferencias estadísticamente significativas $(p<0,05)$

El Tiabendazol tuvo un comportamiento similar a la deltametrina, puesto que hay diferencias estadísticas significativas $(p<0,05)$ entre el clavel fresco $\left(4,67 \mathrm{mg} \cdot \mathrm{kg}^{-1}\right)$, el clavel ensilado a los 0 días $\left(2,23 \mathrm{mg} \cdot \mathrm{kg}^{-1}\right)$ y el clavel ensilado a los 15 días $\left(1,49 \mathrm{mg} \cdot \mathrm{kg}^{-1}\right)$; y no hay diferencia estadística significativa $(p>0,05)$ entre los días 15 y 30 días de fermentación. Los resultados obtenidos en esta prueba indican que, estadísticamente, el dar el ensilaje para alimentación animal el día 15 de fermentación, en la cual los residuos tóxicos de tiabendazol se han rebajado en un mayor porcentaje, sería igual que dar a consumir este producto al día 30 de fermentación. Sin embargo, hay que tener presente que así no haya diferencia estadística significativa, los niveles de residuos tóxicos han disminuido.

\section{Literatura citada}

Alcaldía Municipal de Sora. Información geográfica del municipio de Sora. [en línea]. 2012. URL Disponible en: <http://www.sora-boyaca. gov.co/nuestromunicipio.shtml?apc $=$ mlxx$1-\& m=f>$. [Fecha de acceso 20 de enero de 2012]. 
Aponte, L. y Alfonso, M. (2008). Cultivos protegidos con técnica hidropónica y biológica. Tomo II. Bogotá: Grupo Técnico Aponte.

Cerón, J. J. (1993). El clavel (Dianthus caryophillus) como subproducto en la alimentación, problemática de los residuos de plaguicidas. Tesis doctoral. Universidad de Murcia. España.162 p.

Cerón, J. J. (1994). Subproductos de cultivos de invernadero en la alimentación de la cabra. Estudio del valor nutritivo del subproducto del clavel (Dianthus caryophillus) desecado al sol. URL disponible en: http://www.um.es/ dp-medicina-cirugia-animal/reproduccion/ curriculum/gutierrez.pdf. [Fecha de acceso 26 de febrero de 2012]. 4 p.

Conde, C., Bazante, R. (1990). Digestibilidad in vivo, consumo y evaluación del valor nutritivo de los desechos de clavel (Dianthus caryophillus) en la alimentación de ovinos de engorde. Tesis de grado. Facultad de Medicina Veterinaria y de Zootecnia. Universidad Nacional de Colombia. Bogotá. 256 p.

De-Prado, R., Osuna, M.D. (1999). Resistencia a herbicidas. Detección en campo y laboratorio. Congreso 1999 de la semilla. Logroño, España. pp. 435-440.
Hard, M. y Araya, E. (1996). Evaluación de fungicidas para el combate del Mildeo velloso de la vid en Costa Rica. Universidad de Costa Rica. X Congreso Nacional Agronómico. III Congreso de Fitopatología.

Machin, D. (2006). El uso potencial del ensilaje para la producción animal en la zona tropical, especialmente como una opción para los pequeños campesinos. Depósito de documentos de la FAO. URL disponible en: http://www.fao.org/docrep/005/X8486S/ x8486s07.htm. [Fecha de acceso 15 de febrero de 2012]. 26 p.

Malagón-Mateus, R. (2005). Enfoque de sistemas: una opción para el análisis de las unidades de producción agrícola. Palmira: Universidad Nacional de Colombia. 74 p.

Moorman, T. (1990). Adaptation of Microorganisms in Subsurface Environments. En: Enhanced Biodegradation of Pesticides in the Environment. American Chemical Society (ACS). Washington, USA: 167-180.

Fecha de Recepción: 15 de abril de 2012 Fecha de Aceptación: 4 de mayo de 2013 